\title{
Morpho-histological characterization of truncated leaf syndrome seedlings: an oil palm (E. guineensis Jacq.) somaclonal variant
}

\begin{abstract}
A comparative phenotypic and morpho-histological study was carried out on tissue culturederived truncated leaf syndrome (TLS) and wild-type oil palm seedlings to investigate their phenotypic and morpho-histological differences. On the basis of the percentage of TLS occurence in a clone, the TLS seedlings were categorized into three groups: severe (70Ï $100 \%)$, moderate (40ï 69\%) and mild $(<40 \%)$. Wild and TLS seedlings differ in terms of growth, vigor, leaf size and shape, root number, volume, length as well as the size of shoot apical meristem (SAM). Differences were also found in fresh weight of leaf, root and SAM of TLS in comparison to wild-type seedlings. Depressed and wavy leaf surface, sunken and distorted stomata and coalesced epidermal cells were observed by scanning electron microscopy in TLS seedlings. The size, shape and number of stomata were also different in the TLS leaf compared to the wild type. Longer epidermal cells, depressed epidermal layer, larger sub-epidermal cells and loosely arranged less mesophyll cells were observed in TLS leaf than in wild type. Undifferentiated vascular bundle was found in TLS leaves where metaxylem and phloem were absent and root tips were impaired. The size and leaf primordial arrangement of SAM were remarkably different in TLS compared to wild-type seedlings suggesting that these alterations might be due to smaller SAM. Therefore, further detailed genetic analysis on TLS SAM is needed for clear understanding of TLS occurrence.
\end{abstract}

Keyword: Self-pruning; TLS; Oil palm; Somaclonal variation; Abnormality; Histology; Shoot apical meristem 